\title{
PENSAR CRÍTICO Y PRODUCCIÓN DE CONOCIMIENTO DESDE PRÁCTICAS DE TRANSFORMACIÓN SOCIAL ${ }^{1}$
}

Critical thinking and knowledge production from social transformation practices

\author{
TORRES CARRILLO, Alfonso²
}

"Toda forma de pensamiento crítico debe comenzar por ser una crítica al conocimiento mismo" (Santos, 2003, p. 33)

\begin{abstract}
Resumen: El autor expone los fundamentos, algunos criterios y rasgos metodológicos del quehacer investigativo, enmarcados en la tradición latinoamericana de pensamiento crítico y acción emancipadora. De esta manera presenta, en un primer momento, una concepción ampliada del pensar crítico desde la que esta modalidad investigativa crítica participativa se posiciona, para luego plantear los principios y criterios metodológicos que orientan y configuran estas prácticas investigativas, y finalmente, esboza las principales decisiones y acciones metodológicas que la caracterizan. Esta "investigación social desde el margen" reconoce la presencia de lo subjetivo en todo proceso de construcción de conocimiento y se opta por la exigencia de hacer reflexivas cada una de las decisiones y operaciones investigativas. Esto es, la investigación crítica participativa ha procurado devenir articulada a procesos organizativos comunitarios y a movimientos sociales y ha estado marcada por una identificación con la educación popular.
\end{abstract}

Palabras-clave: Pensar crítico. Investigación crítica participativa. Transformación social.

\begin{abstract}
The author presents the foundations, some criteria and methodological features of the research work, framed in the Latin American tradition of critical thinking and emancipatory action. In this way, the author presents, at first, an expanded conception of critical thinking from which, this participatory critical research modality is positioned. The author poses next the methodological principles and criteria, which guide and configure these research practices, and finally, he outlines the main methodological decisions and actions that it featured. This work called "social research from the margin" recognizes the presence of the subjective in every process of knowledge construction, and the demand to make each one of the decisions and research operations reflective that is chosen. Basically that is, participatory critical research has sought to become linked to community organizational processes and social movements, and also has been marked by identification with popular education.
\end{abstract}

Keywords: Critical thinking. Participatory critical research. Social transformation.

\footnotetext{
${ }^{1}$ Autor convidado para este dossiê da REALIS.

2 Doctor en Estudios Latinoamericanos, profesor de la Universidad Pedagógica Nacional. E-mail: alfonsitorres@gmail.com
} 


\section{Presentación}

En el presente artículo, me propongo exponer los fundamentos, algunos criterios y rasgos metodológicos de mi quehacer investigativo, el cual se enmarca en la tradición latinoamericana de pensamiento crítico y acción emancipadora cuyos orígenes pueden remontarse a las anti-crónica de Guamán Poma de Ayala, pasando por el pensamiento político y pedagógico de Simón Rodríguez y José Martí, hasta llegar a los críticos decoloniales contemporáneos. En particular, me identifico con la perspectiva de investigación participativa, iniciada por Paulo Freire (1970) y Orlando Fals Borda (1970 y 1978), y que, medio siglo después de su formulación inicial, ha tenido importantes desarrollos epistémicos, conceptuales, metodológicos y técnicos, hasta convertirse en la actualidad en una de las tendencias investigativas de mayor reconocimiento e influencia en los estudios sociales contemporáneos ${ }^{3}$.

Paradójicamente, en nuestros medio universitario, dichas perspectivas ocupan un lugar marginal en la formación de los futuros educadores y en las propias investigaciones de los profesores, sea porque seguimos subordinados a las olas teóricas provenientes del Norte epistémico, o porque estos planteamientos críticos resultan incómodos para algunos colegas, más interesados en posicionarse en los rankings institucionales que en cuestionar la institucionalidad, y mucho menos en transformarla. Por ello, he considerado pertinente presentar, en un primer momento, una concepción ampliada de pensar crítico desde la que esta modalidad investigativa se posiciona, para luego plantear los principios y criterios metodológicos que orientan y configuran estas prácticas investigativas, para finalmente, esbozar las principales decisiones y acciones metodológicas que la caracterizan.

Estas consideraciones ya han venido recientemente siendo expuestas - con diferentes énfasis y acentos - en otros escritos, lo que pone de manifiesto el carácter de construcción inacabada que las caracterizan (Torres, 2002; Torres, 2014; Aguilera, González y Torres, 2016; Barragán y Torres, 2017). Reconocemos que la emergencia de

\footnotetext{
${ }^{3}$ Ello se evidenció en la Conferencia ARNA (Action Research Network Association) realizada en Cartagena de Indias en 2017, en la cual participaron más de 1.500 investigadores procedentes de todo el mundo. Ya en 1977 y 1997, Orlando Fals Borda había realizado simposios mundiales con una amplia convocatoria y acogida internacional.
} 
una perspectiva metodológica es el resultado de la confluencia de múltiples aportes y el concurso de diferentes manos, cabezas y corazones; por ello, este es un nuevo pre-texto para continuar en el camino.

\section{Pensar crítico latinoamericano desde diferentes prácticas}

Cuando en el mundo académico escuchamos las expresiones “crítica”, teoría crítica o pensamiento crítico, tendemos automáticamente a asociarlo con una escuela o corriente teórica, en particular con la llamada Escuela Crítica de Frankfurt y a pensadores como Max Horkheimer, Tehodor Adorno, Herbert Marcuse y Jurgen Habermas; algunos recordarán sus filiaciones con la obra de Marx y su reiterada crítica "a todo lo existente". Los más eruditos nos aclararán que la crítica nació como una operación auxiliar de la filología y de la historia (crítica interna y externa de documentos), se elevó en Kant a un ejercicio de cuestionamiento filosófico sobre los límites de la ciencia, la ética y la estética y en los hegelianos de izquierda se orientó a develar cuanto había de alienación y extrañamiento en la religión, el arte y la filosofía misma (Puerta, 2015, p, 125).

También sabemos que, en manos de Carlos Marx, la crítica filosófica pasó a ser una crítica a la propia filosofía y a las nacientes disciplinas sociales (derecho, economía, historia, ciencia política), así como a las ideologías políticas de sus contemporáneos; varios de sus escritos fueron titulados como "crítica" (recordemos su Crítica a la crítica crítica de Proudhon). Pero, sobre todo, Marx (junto con Engels) promovieron una crítica a la sociedad capitalista y su conjunto de instituciones, transitando de crítica intelectual a la crítica social y a la praxis revolucionaria.

A partir de sus seminales planteamientos, a lo largo del siglo XX, el marxismo se fue erigiendo como la corriente crítica por excelencia, frente a otras posturas epistemológicas, teóricas y políticas, consideradas tradicionales, conservadoras o "burguesas". Es el caso ya mencionado de la Escuela de Frankfurt (que también asumen el psicoanálisis en clave crítica), y también el de otros pensadores como Antonio Gramsci, George Luckas, Karl Korsch, Ernst Bloch, Henri Lefebvre, Alain Badiou, Jacques Ranciere y Slavoj Zizek, quienes han retomado, renovado y ampliado esta tradición crítica. 
Pero si entendemos la crítica "como una acción que permite la emergencia de una política y una ética de la vida y como una perspectiva para analizar las complejas relaciones entre la vida y el poder" (Walzer, 1993), o como "el movimiento por el cual el sujeto se atribuye el derecho de interrogar la verdad acerca de sus efectos de poder y al poder acerca de sus discursos de verdad, la crítica será el arte de la inservidumbre voluntaria, de la indocilidad reflexiva" (Foucault (2006); no existe un modo único o superior de pensar crítico; por lo menos, en la tradición reciente de la Slavojfilosofía, varias corrientes se han asumido como críticas; basta con traer la famosa alusión de Ricoeur (2003) a los "maestros de la sospecha" para referirse a Marx, Nietzsche y Freud, portadores de un escepticismo crítico, desde el cual las cosas no son como se muestran y hay que desenmascararlas para develar su lado oscuro: intereses de clase, voluntad de poder e inconsciente reprimido. Es por ello que también podemos incorporar a esta amplia corriente del pensar crítico a otros autores, fundamentados en la propia crítica a ciertos rasgos y planteamientos del marxismo y que incorporan otras perspectivas de bordaje del poder y el conocer, como es el caso de Michel Foucault, Giles Deleuze y Felix Guattari.

En esta misma dirección, podemos ir más allá y reconocer que la crítica social no es exclusiva o consecuencia de la invención filosófica y político, sino que está presente en diferentes escenarios, prácticas y entre diversos actores modernos y contemporáneos. En efecto, el cuestionamiento frente a las verdades y los poderes establecidos se ha dado tanto desde la filosofía como desde las ciencias sociales y otras prácticas intelectuales, estéticas, sociales y culturales; en el primer ámbito, es reconocida toda una corriente de investigación social crítica, desde el Norte, que se sustenta en los planteamientos de la escuela de Frankfurt, en particular de la idea de "ciencia social crítica" de Habermas, que más allá de explicar o comprender la sociedad, apunta a su emancipación; en esta perspectiva encontramos, por ejemplo, investigadores educativos como Carr y Kemmis (1988), quienes plantean una investigación educativa crítica, o Henry Giroux, Apple y Peter McLaren, referentes norteamericanos de la pedagogía crítica. Corrientes similares las encontramos en otras ciencias sociales y campos profesionales como la historia, la geografía, la sociología, la comunicación y el trabajo social. 
También desde el Sur global han emergido propuestas críticas de ciencia e investigación social que, a partir de la crítica al carácter, imperial, colonial y capitalista de la ciencia social que se impuso desde el Norte, han construido pensamientos, teorías y prácticas investigativas que pretenden contribuir a la emancipación intelectual, cultural y política de nuestros continentes "periféricos". Es el caso de los estudios post-coloniales (Franz Fanon, Edward Said, Homi Bhabha), estudios subalternos en la India (Ranajit Guha, Gayatri Spivak, Aijaz Ahmad) y decoloniales en América Latina (Riverai), los cuales han puesto en evidencia la pervivencia de las visiones e imaginarios coloniales una vez finalizada la dominación británica en la India y...?.

En América Latina también existe una amplia tradición de pensamiento social crítico; en unos casos, de inspiración marxista, representado en intelectuales como José Carlos Mariátegui, Florestán Fernández, Ruy Mauro Marini, Franz Hinkelammert, Helio Gallardo y Renán Vega; en otros, en diálogo con otros referentes críticos (intelectuales, políticos, éticos y culturales) como Camilo Torres Restrepo, Pablo González Casanova, Enrique Dussel, Orlando Fals Borda, Aníbal Quijano, Hugo Zemelman y Catherine Walsh. Estos pensadores, además de cuestionar las teorías y prácticas de las ciencias sociales hegemónicas (desarrollistas, imperialistas, eurocéntricas, coloniales), abogan por la necesidad de unas ciencias sociales latinoamericanas propias, críticas, decoloniales e interepistémicas.

Cabe destacar, en este sentido, a Orlando Fals Borda, quien no solo contribuyó a la crítica al colonialismo intelectual de la ciencia social institucionalizada (1970), sino que fue más allá, al plantear la posibilidad de una "ciencia popular", que finalmente materializó en su propuesta metodológica de la Investigación Acción Participativa, que pretende producir conocimiento comprometido con y desde las luchas populares, involucrando como sujetos de conocimiento a los actores de dichas prácticas; su obra es en la actualidad un referente de otras metodologías y formas de producción conocimiento alternativas como la recuperación colectiva de la historia, la sistematización de experiencias, la auto-indagación crítica (Avesol, 2013) , la pesquisa ação (Thiollent, 2016) y la pesquisa participante (Brandão, 1981 y 2015).

Si reconocemos que la crítica no se refiere solo a la problematización de las diferentes articulaciones entre conocimiento y poder, sino también a la visibilización y 
cuestionamiento de otras condiciones y situaciones de dominación, el panorama de prácticas críticas se amplía; si, como plantea Butler (2008, p. 141), "la crítica es siempre crítica a una práctica, discurso, episteme o institución, instituidos”, también está presente en otras acciones culturales, artísticas, educativas, comunicativas y sociales que controvierten e impugnan el statu quo. Es el caso, en América Latina, de un conjunto de discursos y prácticas alternativas, tales como la educación popular, la comunicación alternativa, el derecho alternativo y la teología de la liberación, que en las últimas décadas, se han convertido en corrientes de pensamiento y movimientos de gran influencia en una amplia gama de procesos organizativos y movimientos sociales a nivel mundial.

Estos últimos también han sido referente y lugar de emergencia de pensar y actuar críticos, aportando, a su vez, a la configuración de las corrientes filosóficas, las ciencias sociales y las prácticas intelectuales críticas ya mencionadas. En efecto, han sido los diferentes movimientos sociales contemporáneos los que han inspirado a pensadores críticos como Carlos Marx y a perspectivas como los diferentes feminismos, el ambientalismo, la ecología política y la interculturalidad crítica; así mismo, como acabo de señalarlo, han sido las luchas y procesos organizativos populares el nicho privilegiado de iluminación y recepción de las prácticas educativas, comunicativas y culturales alternativas.

En un sentido aún más amplio, podemos reconocer como críticas, las actitudes, posiciones y acciones de colectivos sociales que resisten a situaciones o condiciones que interpretan como indignas e injustas, y frente a las cuales despliegan un conjunto de sentires, saberes y sabidurías portadores de sentidos críticos. Estos modos de sentir/pensar/actuar crítico no son meramente "reactivos" a las políticas hegemónicas; son también creativas, pues generan lugares de enunciación otros, desde los cuales se cuestionan los poderes y verdades establecidos y se promueven otras relaciones y sentidos de vida.

Estos sentires, saberes y comprensiones no se manifiestan siempre en forma de sistemas discursivos, sino de ideas poco articuladas, de expresiones simbólicas y difusas prácticas culturales propias de los dominados (Scott, 2000). En esa perspectiva, me identifico con lo planteado por Oscar Useche (2016) acerca de 
El pensamiento crítico de resistencia se propone a aportar a una analítica del acontecimiento resistente fundado en la práctica de los excluidos que buscan afirmar su propio poder molecular basado en la diferencia. Esto implica interrogar las prácticas y los discursos de lo(a)s resistentes sociales y examinar los trayectos por los que fluyen sus luchas en los límites, siempre inciertos, de la reconstitución de sus condiciones de existencia social; desde allí se cuestiona al conjunto de relaciones sociales y a las diversas formas de dominación social (Useche, 2016, p. 22)

Estos saberes y pensares críticos "desde abajo y desde el Sur" poseen una larga historia en América Latina. Se remontan al momento mismo de la invasión europea, pasando por la colonia y el periodo de independencia, con la aparición de una conciencia americana (Roig, 2004), el ensayismo crítico de la segunda mitad del siglo XIX y primera del XX, la emergencia de las ciencias sociales críticas y otras prácticas "alternativas" desde las décadas de 1970 y 1980. En la actualidad, asistimos a un renacer del pensamiento crítico, expresado en algunas perspectivas emergentes como los planteamientos decoloniales, el pensar epistémico y las epistemologías del Sur.

Un proyecto intelectual que se ha venido configurando y cobrando influencia en el campo intelectual crítico en las 2 últimas décadas es el de las teorías de-coloniales (Mignolo, 2006; Grosfoguel y Romero, 2009), las cuales, a partir de una radical crítica al proyecto occidental moderno y colonial, encuentra en las prácticas y reflexiones situadas desde la resistencia y re-existencia de los pueblos, un lugar epistémico y político para cuestionar y subvertir la racionalidad y el poder colonial en diferentes prácticas de saber, poder y ser, alter-nativas (Walsh, 2002; Albán, 2010).

Por otro lado, están los planteamientos del pensador chileno Hugo Zemelman, quien hace un llamado a transgredir los límites de la racionalidad de la ciencia, dado que ésta no permite dar cuenta de la complejidad y dinamismo de la vida social. El reconocimiento de la historicidad e indeterminación de la realidad social, así como el papel de los sujetos y la subjetividad social en su transformación, desbordan el conocimiento científico atrapado en la razón analítica y a la lógica del objeto, desde corpus teóricos preestablecidos y lenguajes cerrados (Zemelman, 2005).

Para este pensador chileno, conocer la realidad desde opciones de construcción de realidad exige una apertura gnoseológica que permita "captar" su riqueza y multiplicidad, a la vez que incorpore otras dimensiones no cognitivas del sujeto, como la voluntad y la emocionalidad. Esta racionalidad crítica, que Zemelman nombra pensar epistémico 
(Zemelman, 2007), implica un posicionamiento de los sujetos frente a sus circunstancias, apropiación de la realidad desde diferentes ángulos y lenguajes y reconocimiento de sus posibilidades de desenvolvimiento. En fin, es desde la necesidad de construcción de realidad y de conciencia histórica que para los sujetos cobra sentido la investigación social.

También encontramos el monumental trabajo de Boaventura de Sousa Santos, quien, a partir de un cuestionamiento a la ciencia social moderna (indolente, abismal, de las ausencias), busca refundar el pensamiento crítico desde el reconocimiento de los sentidos, saberes y visones de futuro presentes en los movimientos sociales y las prácticas emancipadoras. Para Santos, existe en la actualidad una crisis general de las ciencias sociales constituidas en la modernidad, que se expresa en su imposibilidad de comprender y decir "algo nuevo" frente a las problemáticas más significativas del mundo actual. Desde el Sur y desde los movimientos y organizaciones sociales alternativos, "no es simplemente un conocimiento nuevo el que necesitamos; necesitamos un nuevo modo de producción de conocimiento. No necesitamos alternativas, necesitamos un pensamiento alternativo de las alternativas" (Santos, 2005, p.16).

Finalmente, desde una perspectiva más modesta, están nuestras reflexiones acerca de la "investigación social desde el margen" o liminal, que hacen referencia al conjunto de prácticas de producción de conocimientos que, articuladas a procesos organizativos y a movimientos sociales, desarrollan colectivos de investigadores comprometidos con propósitos políticos y epistémicos emancipadores y que no tienen un "lugar" específico en el campo de las disciplinas sociales o en la institucionalidad académica; más bien, se sitúan en los bordes de éstas, o en sus intersticios, en la medida en que asumen una actitud nómada, móvil, fluida, entre las dinámicas movimientistas y los recorridos conceptuales y metodológicos generados, tanto en los estudios sociales críticos, como en las prácticas investigativas alternativas. (Torres, 2004 y 2008; Valiente, Godfrid y Bertea, 2017).

Esta investigación liminal sería equivalente a lo que otros autores han denominado como "investigación de frontera" (Anzaldúa, Mignolo), "razonamiento de umbral" (Zemelman), ya que, al estar o transcurrir por fuera de las epistemes instituidas, permiten ver, hacer y producir prácticas y conocimientos que desbordan los órdenes de saber y poder instituidos. Es el caso de las diferentes modalidades de investigación participativa, 
tales como la Investigación militante y la Investigación Acción Participativa, la recuperación colectiva de historias locales y organizativas, y la Sistematización de prácticas desde la experiencia de sus actores. En éstas modalidades investigativas, las iniciativas, las preguntas, los diseños y estrategias metodológicas, así como la interpretación y comunicación de sus resultados, se construyen siempre en un diálogo entre colectivos u organizaciones de base e investigadores sociales, generando sentidos, conocimientos, prácticas y subjetividades instituyentes, muchas veces alternativos.

\section{Criterios metodológicos que orientan la producción de conocimiento ligada a la acción colectiva}

En mi caso, la opción por la perspectiva de investigación social crítica ha estado asociada a mi experiencia como educador popular, historiador e investigador social; experiencia asociada a prácticas como militante social y educativo y como acompañante y formador de colectivos y organizaciones sociales, así como a prácticas de producción de conocimiento desde y para la acción colectiva y de construcción de metodologías que potencian dichas prácticas, tales como la recuperación colectiva de la historia y la sistematización de prácticas de transformación social. En este caminar, he tenido el privilegio de trabajar y recibir influencia (personal y como lector) de Paulo Freire, Orlando Fals Borda, Jesús Martín-Barbero, Mario Peresson, Hugo Zemelman, Jesús Ibáñez, Michel de Certeau, Cornelius Castoriadis y Boaventura de Sousa Santos.

Desde mi participación en la Coordinadora Distrital de Educación Popular (en los 80s), en el equipo de Dimensión Educativa (en los 90s), en el colectivo de educadores e investigadores populares de la Universidad Pedagógica Nacional (de los 90s a hoy), en el Programa Latinoamericano de Sistematización de experiencias del CEAAL (1996 a 2016) y en algunos Grupos de Trabajo de CLACSO (2010 a hoy), hemos venido construyendo cierta manera de entender y hacer investigación con colectivos de educadores y organizaciones populares. Esta búsqueda, aún abierta, nos ha llevado a nombrarla de diferentes modos: "investigar en comunidad” (Torres, 1995 y 1996), “investigación interpretativa crítica” (Torres, 1996 y 1997; Barragán y Torres, 2017), “investigación 
crítica" (2000 y 2014), "investigación cualitativa crítica" (Torres, 2003) e "Investigación desde el margen" (Torres, 2004 y 2008).

Dicha perspectiva, epistémica y metodológica, asume la investigación como una práctica colectiva de producción de conocimiento, articulada a procesos organizativos y movimientos sociales, que se propone comprender y fortalecer su capacidad de resistencia y generación de alternativas al capitalismo, así como contribuir a la producción de subjetividades y de vínculos comunitarios y a la transformación de realidades y sujetos desde una perspectiva crítica y emancipadora. Por ello es que en este texto decidimos nombrarla como producción de conocimiento en prácticas de transformación social. Allí caben diferentes iniciativas intencionales de resistencia, alteración, subversión, acción colectiva y construcción de alternativas, tanto a nivel macro social como micro social, frente a diferentes situaciones, relaciones y condiciones de dominación (económica, política, cultural, de género, etc.).

Desde esta concepción y práctica investigativa hemos venido elaborando un conjunto de principios epistémicos y criterios metodológicos que fundamentan y orientan nuestras investigaciones, las cuales discutimos y re-creamos permanentemente. A continuación, las sintetizamos:

1. Posicionamiento crítico frente a las concepciones hegemónicas ${ }^{4}$ de ciencia e investigación social predominantes en el mundo académico, generalmente subordinadas a las epistemes eurocéntricas, modernas, patriarcales, colonialistas y coloniales que les subyacen; así mismo permanecen ancladas en los viejos y nuevos positivismos, que suponen la existencia de un único método científico y desconocen otras formas de producción de conocimiento. Este cuestionamiento a la ciencia predominante se nutre de diferentes perspectivas tales como el marxismo, feminismo y las teorías de-coloniales. Desde nuestra posición, afirmamos con Santos (2006 y 2013) que esto modos de investigar hegemónicos, desprecian, invisibilizan o reprimen los saberes tradicionales,

\footnotetext{
${ }^{4}$ Asumo la concepción de hegemonía planteada por Gramsci, según la cual, los sectores dominantes de la sociedad, garantizan su poder a través de mecanismos coercitivos, sino culturales e intelectuales, asumiendo también su rol de clases dirigentes. De este modo, subordinan a grupos sociales como los intelectuales, que termina por crear un "bloque ideológico", que liga a las capas intelectuales a los proyectos de las clases dirigentes. (Portielli, 1985, p. 71)
} 
ancestrales y populares; la mayoría de las veces, solo acuden a estas poblaciones de base como "fuentes de información", llevando a cabo un verdadero "extractivismo cognitivo".

2. Investigación localizada. Frente al universalismo propugnado por la ciencia clásica, que abstrae los contextos históricos y culturales desde y sobre los cuales se produce el conocimiento, nuestras prácticas de producción de conocimiento reivindican su radical contextualidad. Propugnamos por un conocimiento situado y articulado a las necesidades y desafíos de las realidades históricas, políticas, sociales y culturales en que se localizan. Esta posición corresponde a lo que Hugo Zemelman plantea como "exigencia de historicidad", punto de partida ineludible en cualquier investigación social, y Dana Haraway (1995) nombra como "investigación situada".

3. Identificación con opciones éticas y políticas de transformación social inspiradas en visiones de futuro alternativas al orden imperante; esta orientación emancipadora ha sido nombrada de diferentes maneras, según el contexto histórico y cultural, tales como el "todavía no" (Bloch), "inédito viable" (Freire), socialismo raizal (Fals Borda), "heterotopías" (Foucault) y "otros mundos posibles" (Foro Social Mundial). Las maneras de entender su realización también han variado en los tiempos contemporáneos: desde las visiones revolucionarias inspiradas en la imagen de una casa que se incendia y sobre sus ruinas se construye una nueva, hasta otras que buscan realizarse desde el aquí y el ahora, a través de diferentes prácticas culturales, intelectuales, educativas, investigativas, comunicativas y estéticas de carácter instituyente.

4. Compromiso y articulación con luchas sociales y otros procesos de acción colectiva agenciadas por sectores sociales populares y subalternos. En la etapa fundacional de las metodologías participativas, eran los profesionales o investigadores quienes decidían involucrarse o insertarse en los procesos organizativos y movimientos sociales (Fals Borda, 1970); en momentos posteriores, fueron las Organizaciones civiles de apoyo a dichos procesos quienes asumieron el rol de "investigadores orgánicos"; también se fue haciendo frecuente que grupos de investigación vinculados a universidades asuman este mismo rol, a 
través de acuerdos con dichos actores sociales. En la actualidad, son las propias organizaciones y movimientos sociales quienes deciden la realización de sus propias investigaciones, incluso, generando instancias permanentes de producción de conocimiento, las cuales son quienes deciden con quienes realizar sus investigaciones.

5. Una producción de conocimiento "nómada" o "liminal" que no se define ni se subordina a la lógica institucional de la investigación disciplinar (Torres, 2008 y 2014). No por capricho o moda académica, sino por la propia naturaleza de los sentidos que la animan y los problemas de los que se ocupa: su interés emancipador y su intención de comprender para transformar procesos y prácticas sociales singulares impone abordajes que atraviesan fronteras institucionales, epistemológicas y metodológicas. De este modo, las investigaciones que realizamos casi siempre se sitúan entre los mundos académico y popular, entre la producción de conocimiento y la acción política; estar en movimiento $\mathrm{y}$ en los intersticios permite ver y hacer cosas inimaginables e imposibles desde los "centros" de la institucionalidad académica y científica.

6. Promueve la participación de la gente común y corriente, "no especialistas" (integrantes y dirigentes de organizaciones y movimientos populares, educadores, activistas) en la producción de conocimiento; ello se expresa, no solo en las operaciones investigativa sino principalmente en la toma de decisiones estratégicas del proceso metodológico, tales como por qué y para qué iniciar un proyecto investigativo, cuáles serán las preguntas y problemas que lo orientarán, cuáles las estrategias de producción y análisis de los datos y en la interpretación global de resultados. Para ello, generalmente se forma un equipo responsable de la investigación, conformado por personas de las organizaciones o movimiento y quienes asumen el rol de acompañantes o asesores.

7. Asume las prácticas investigativas como experiencia de formación como sujetos de conocimiento y de pensamiento. En concordancia con la apuesta de contribuir a que los sectores populares se vayan conformando como sujetos sociales autónomos, críticos y propositivos, desde estas prácticas investigativas se pretende que quienes participan en ellas adquieran y afiancen sus capacidades de 
pensamiento y conciencia crítica en la apropiación de enfoques y estrategias metodológicas y de herramientas investigativas prácticas.

8. Promueve el diálogo entre los diferentes saberes de los que son portadores los actores participantes de cada investigación. Frente a la actitud monológica y arrogante del conocimiento científico predominante, que desconoce los saberes que no obedecen a su lógica empírica o los utiliza solo para "corroborar" sus teorías, las investigaciones participativas favorecen la confluencia e interacción dialógica entre diferentes formas de pensar, conocer, valorar y sentir. Por ello es que acogemos los aportes provenientes de la educación popular acerca del diálogo de saberes y el planteamiento de Santos (2006) acerca de la ecología de saberes.

Reconocemos la pluralidad de dimensiones y sentidos que configuran los procesos comunitarios, las organizaciones sociales y la acción colectiva; por ello, dichas realidades no pueden quedar atrapadas en una sola racionalidad o sistema cultural. En consecuencia, nuestras investigaciones procuran la confluencia - casi siempre conflictiva - de diferentes formas de pensar, interpretar y narrar la realidad. Partiendo de los saberes, lenguajes y formas de comprensión propias de los actores sociales participantes, el abordaje de las preguntas que orientan las investigaciones también involucra los conocimientos y procedimientos provenientes del campo científico, de las prácticas artísticas y de las sabidurías ancestrales y populares; ello permite cuestionar y ampliar la mirada del colectivo y generar nuevas lecturas sobre las problemáticas investigadas.

9. A diferencia con la racionalidad predominante en el mundo académico que le da prioridad a la teoría sobre la práctica y con las propuestas que ven esta solo como una aplicación de aquella, en nuestras investigaciones se reconocen los conocimientos que provienen de las propias prácticas sociales, los que se generan en las luchas y movimientos sociales. Es desde dichas prácticas que se dialoga con las perspectivas teóricas y conceptualizaciones provenientes de diferentes campos de conocimiento; así mismo, se procura que los conocimientos generados en las investigaciones retornen a las prácticas sociales de las que surgieron, para potenciarlas y transformarlas. 
10. Relación y uso crítico de la teoría. En la medida en que privilegiamos la historicidad y singularidad de los procesos y emergencias sociales y no la aplicación de marcos teóricos previos, partimos de reconocer los factores y sentidos que estructuran los problemas de estudio y la manera como los sujetos categorizan e interpretan dichas realidades. Una vez hecho el reconocimiento de estas lógicas y significados, acudimos a los referentes conceptuales y teóricos que consideramos pertinentes para profundizar o problematizar la lectura inicial de los hallazgos; de este modo, el uso que damos a la teoría no es deductivo (adecuar una realidad a un marco interpretativo previo) ni inductivo ("descubrir" las teorías implícitas), sino transductivo, es decir, provoca una dialéctica entre la comprensión de lo particular y la interpretación en marcos más generales, lo que permite la creación conceptual y la comunicación con otras realidades similares. Asumimos las teorías como formas de racionalidad surgidas en contextos epistémicos e históricos específicos con la potencialidad de recrearse para interpretar nuevas realidades (Zemelman, 2005).

11. Nuestras investigaciones también asumen como principio la flexibilidad y la creatividad metodológica. Frente a la rigidez y linealidad de la racionalidad instrumental de la investigación que privilegia diseños rígidos, estrategias y técnicas estandarizadas, desde nuestra perspectiva, las metodologías son construcciones que deben ser asumidas de una manera crítica y creativa. Ello ha posibilitado que en nuestras investigaciones haya una preocupación permanente por adecuar e innovar las estrategias y procedimientos empleados, en función de la singularidad de los sentidos, sujetos y preguntas que definen cada proyecto; así, por ejemplo, en la recuperación colectiva de la historia hemos creado unos "dispositivos de activación de memoria" (paseos del recuerdo, museos comunitarios, tertulias) que a la vez que provocan relatos sobre los temas, afianzan los vínculos y los sentidos de pertenencia colectivos (Cendales y Torres, 2001a).

Así mismo, procuramos ser abiertos e imaginativos en el uso de diferentes referentes conceptuales, categorías, estrategias, lenguajes, técnicas dentro de las prácticas investigativas. No por un afán de "innovación" sino frente a la necesidad de dar cuenta de la singularidad de nuestras realidades y prácticas, y en función de 
potenciar los sujetos y las subjetividades que hacen posible la construcción de conocimiento y la transformación de prácticas y realidades sociales.

12. Reflexividad. Frente a la perspectiva positivista, en la que se asume que la posición del investigador es la de observador externo a la población "objeto" de investigación es lo que garantiza objetividad, desde nuestra manera de producir conocimiento con la gente asumimos que lo que hay que promover es que ésta se convierta en sujeto de conocimiento; transformados en colectivos auto observadores, los actores sociales problematizan e indagan su realidad en diálogo con otros actores. Así, los actores/investigadores reflexionan permanentemente sobre las implicaciones de sus posiciones y visiones en cada uno de los momentos y decisiones investigativas. Al reconocer esta presencia de lo subjetivo en todo proceso de construcción de conocimiento, se opta por la exigencia de hacer reflexivas cada una de las decisiones y operaciones investigativas, así como la generación y recreación de criterios que orienten los procesos investigativos. Esta reflexividad permanente posibilita lo que Jesús Ibáñez (1998) denomina "investigación social de segundo orden", la cual implica que, en nuestras investigaciones, debemos estar atentos, durante todo el proceso, tanto a dimensiones epistemológicas (construcción de los problemas de estudio, reconstrucción empírica, análisis, interpretación y teorización), como a otras dimensiones como lo político (participación, democratización, articulación a procesos organizativos, acciones colectivas que se derivan, etc.) y lo pedagógico (formación de participantes, retroalimentación de los avances, comunicación de resultados, atención al lenguaje analítico y narrativo, etc.).

\section{Momentos y decisiones propias de las investigaciones críticas y participativas.}

Los autores fundadores de esta tradición participativa y crítica latinoamericana insistieron en los presupuestos epistémicos y políticos que la sustentan, así como en los criterios metodológicos que la orientan; no centraron su atención en plantear 
prescripciones acerca de los procesos investigativos. Aunque compartimos esta posición, consideramos importante socializar los itinerarios comunes en este tipo de prácticas de producción e conocimiento; por ello, a partir de una reconstrucción "a posteriori" de las secuencias y decisiones tomadas en nuestras investigaciones, podemos identificar algunos hitos comunes a nuestros itinerarios investigativos ${ }^{5}$. Estos no deben ser entendidos como "pasos" de un proceso lineal, sino como nudos problemáticos que, si bien tienen una mayor importancia en un momento determinado, deben tenerse en cuenta a lo largo de toda la investigación.

\subsection{El punto de partida: Opciones, confianzas y consensos con los grupos u organizaciones sociales}

La iniciativa de nuestras investigaciones puede tener diferentes fuentes: el interés del colectivo que agencia una organización o movimiento por comprender mejor su práctica o una realidad que los problematiza, la decisión de nuestro colectivo de investigación por indagar un asunto que resulta relevante dentro de un campo problemático en el que venimos trabajando, la convocatoria o invitación de una instancia de cooperación o espacio de articulación más amplio para abordar una temática de interés general.

En todos los casos, es necesario conversar y llegar a acuerdos sobre la pertinencia y relevancia de la investigación para los actores que se van a involucrar y definir las finalidades que la orientarán. Por lo general, en las razones que justifican nuestras investigaciones se conjugan la preocupación de los colectivos por analizar críticamente

\footnotetext{
${ }^{5}$ Algunos de los proyectos de investigación desarrollados siguiendo estas orientaciones son: Discursos, prácticas y actores de la educación popular en Colombia durante la década de los ochenta (1995-1996); Organizaciones populares, identidades locales y ciudadanía en Bogotá (2001-2002); Saberes y prácticas pedagógicas en organizaciones populares (2003-2004); Formación de pensamiento crítico en procesos educativos populares (2005-2006); Educación en movimientos sociales de América Latina (2011-2012); Auto-indagación crítica de la historia de AVESOL: 36 años de resistencia popular en el suroriente bogotano (2012 a 2013); Formación de subjetividades y comunidad en organizaciones locales en Colombia (20132014); Construyendo memoria colectiva de la Universidad pedagógica nacional: tres propuestas de educación comunitaria (2018 - 2019).
} 
su práctica con nuestro interés por ampliar nuestra comprensión de este tipo de procesos sociales y de acciones colectivas, con el fin de fortalecer su potencial emancipador.

Por ejemplo, en el proyecto "Formación de subjetividades, sentidos de comunidad en procesos organizativos locales", vimos la importancia de abordar cómo las organizaciones desde sus procesos formativos potencian la constitución de subjetividades políticas. A la vez, algunas organizaciones se han acercado al grupo para solicitar apoyo porque desean realizar alguna investigación que les ayude a comprender en qué medida sus prácticas mantenían su carácter crítico y autónomo.

También es común que antes de iniciar el trabajo práctico de la investigación, se busque generar las condiciones logísticas y se elabore el plan de trabajo donde se especifican las actividades, los tiempos, las responsabilidades y los recursos. Ello implica armonizar los tiempos y procesos de la universidad o institución que respalda la investigación con los tiempos y prácticas de las organizaciones, en particular, las referidas a las condiciones y procedimientos administrativos para la ejecución de los recursos.

\subsection{Problematizar y preguntar (nos)}

Establecidos estos acuerdos y condiciones, se procede a definir los interrogantes que orientarán la búsqueda; a diferencia de las investigaciones convencionales en las que estos provienen de los marcos teóricos y analíticos de los investigadores, en las nuestras la decisión es más política que teórica: surgen del reconocimiento de las cuestiones "vitales" que se está planteando la organización en el momento; es decir, es la lectura crítica de su presente la que posibilita la elaboración de las preguntas en torno a las cuales se organizará la investigación. Por ejemplo, en el ejercicio de auto-indagación recientemente realizada con la Asociación Vecinos Solidarios (AVESOL), una de las inquietudes era ¿En qué medida su creciente vinculación como contratistas de la administración distrital les había hecho perder su autonomía y carácter alternativo; en términos concisos lo expresaron así: ¿Está la lógica contractual desplazando la lógica comunitaria? Otras inquietudes eran ¿Cuáles habían sido las acciones que habían posicionado a la organización frente a la comunidad local? 
A partir de estas preguntas centrales o ejes de indagación se elaboran preguntas específicas que orienten la reconstrucción descriptiva de la experiencia y el balance interpretativo de los hallazgos y aprendizajes. En la investigación con AVESOL, se definió que en el abordaje de cada hito significativo relacionado con la pregunta principal, se haría desde las siguientes preguntas: ¿Cómo surgieron estas iniciativas? ¿Cómo se dio el proceso de construcción y ejecución? ¿Cuáles acciones se desarrollaron y de qué manera? ¿Qué factores incidieron en las acciones? ¿Cómo participó la comunidad? ¿Cuáles fueron los resultados? ¿Cómo se afectó la organización? ¿Qué aprendizajes se generaron?

Estas preguntas y ejes temáticos iniciales, se pueden modificar a lo largo del proceso investigativo, lo cual exige una sensibilidad para identificar preguntas y categorías emergentes. Por ejemplo, en la sistematización de una experiencia organizativa de mujeres, los temas iniciales estaban referidos a sus relaciones con el contexto barrial, a sus dinámicas organizativas internas y a la construcción de su identidad como mujeres; sin embargo, cuando se realizaban las entrevistas y los grupos de discusión, fue emergiendo como relevante el hecho de que su vinculación a la organización local había transformando sus relaciones de pareja. Esta preocupación emergente adquirió centralidad en el desarrollo posterior de dicha investigación.

Definido el qué se va a investigar, también se acuerda cuál será la estrategia metodológica más pertinente; unas veces puede ser la Investigación Acción Participativa, otras la Recuperación colectiva de la historia o la Sistematización de experiencias, una combinación de las mismas o la creación de una nueva estrategia, como lo fue la "autoindagación crítica" para el caso de AVESOL. En todo caso, la decisión pasa por un primer acercamiento a estas metodologías y una vez tomada la decisión, se realiza un taller para profundizar en la perspectiva y familiarizarse con el proceso metodológico global y sus implicaciones prácticas.

\subsection{Con-formación del equipo investigador}

Aunque en el momento anterior se avanza en la definición de quiénes van a hacer parte del equipo investigador, ahora se definen los integrantes de dicho equipo, tanto 
desde el grupo de investigación como desde las organizaciones sociales; para estas últimas, en unas ocasiones la decisión es voluntaria (interés por investigarse o aprender), en otras es una tarea asignada por la dirección (en una investigación con una Cooperativa de campesinos, la junta directiva responsabilizó de la tarea al Comité de educación).

Es este equipo el que asumirá la orientación y desarrollo práctico de la investigación así se estén socializando sus avances con los dirigentes y demás integrantes de la organización. Es este colectivo el que afina la justificación, los objetivos, las preguntas y la estrategia metodológica, los cuales se redactan como un proyecto. Es también con este equipo que se realiza una formación metodológica más intensa, generalmente a través de encuentros periódicos y talleres que proveen de criterios y herramientas para el desarrollo de cada una de las fases de la investigación (reconstrucción histórica, análisis e interpretación, redacción y socialización de resultados) y para apropiarse de los referentes conceptuales que ayudarán a dar una comprensión más profunda de las temáticas emergentes.

\subsection{Reconstrucción descriptiva y narrativa de las experiencias y los procesos organizativos}

Este momento busca producir un relato consensuado de las trayectorias organizativas, en particular de los procesos o hitos significativos con relación a las preguntas que orientan la investigación. Generalmente procedemos a establecer cuáles fuentes nos pueden proveer de información y narrativas sobre las mismas, a cuáles técnicas podemos acudir y cuáles instrumentos para recolectar y registrar los datos.

Una de las primeras actividades es la reconstrucción temporal del proceso, para reconocer sus continuidades y rupturas, así como los hitos y etapas más significativos, para así hacer una primera periodización que nos permita organizar temporalmente la tematización que se deriva de las preguntas. Esta periodización puede hacerse a partir del testimonio de alguna persona que tenga la visión de conjunto o de un ejercicio de construir colectivamente el camino recorrido por la organización en relación a los temas de interés. 
Luego se procede a la reconstrucción descriptiva de los procesos de la organización a partir de las preguntas, las fuentes y las técnicas acordadas. Por lo general, se consultan documentos, se realizan entrevistas y recogen testimonios, se realizan talleres y grupos de discusión; una vez registrada la información provocada por cada técnica, se categoriza y se organiza en torno a la periodización. Finalmente, se redacta un texto que presenta la reconstrucción narrativa y descriptiva del proceso histórico y de los ejes temáticos que visibilice las diferentes voces y miradas; dicha versión se socializa entre los demás integrantes de la organización y ajustada según sus aportes.

\subsection{Interpretar la lógica y los sentidos que configuran el problema}

El proceso de análisis debe considerarse como una actividad reflexiva que influye en el procesamiento de la información y en la redacción de los balances parciales; no obstante, conviene que en todo proceso de investigación exista un espacio-tiempo definido para revisar detenidamente la información acopiada a partir de lo cual realizar los primeros balances. A este momento es a lo que se denomina como "análisis de la información", el cual podría definirse como un conjunto de operaciones empírico conceptuales mediante las que se construyen y procesan los datos a través de un proceso de ordenación, clasificación, categorización y relacionamiento de la información.

En nuestros trabajos, el proceso de análisis pasa por la construcción de categorías (unidades temáticas) que facilitan la agrupación de información. Las categorías son construcciones de sentido que sirven para clasificar y agrupar datos con atributos o propiedades comunes, permiten pasar de una mirada global (la información recogida archivos, entrevistas, conversatorios, etc.) a una más focalizada que facilite la realización de otros procesos analíticos como la construcción de matrices, cuadros, diagramas y esquemas.

Una vez se tiene organizada la información a partir de matrices, cuadros de relación y/o esquemas, se inicia la reconstrucción global de los hallazgos, que a partir de su interpretación, puedan dar respuesta a las principales preguntas y objetivos del estudio. Dicha comprensión es uno de las actividades más complejas, porque implica 
disponer de referentes conceptuales que sean pertinentes a la singularidad de los hallazgos.

La meta de este momento es producir un texto analítico e interpretativo que evidencie una lectura crítica de la problemática estudiada, que a la vez reconozca el punto de vista de los sujetos involucrados, las incidencias del contexto, la interpretación critica de los procesos y categorías emergentes. Dicho balance interpretativo es nuevamente presentado al colectivo para su discusión y ajuste.

\subsection{El punto de llegada: La escritura y la socializa-acción}

¿Cómo se traduce lo encontrado en términos de resultados de conocimiento y para la socialización? La idea es construir una interpretación que le permita a los sujetos reconocerse en los análisis y asumir los retos que depara la lectura crítica de la experiencia organizativa. Este es un desafío importante porque se trata de condensar el conocimiento construido de manera sintética y didáctica; por un lado, la descripción densa de la experiencia, por otro, su análisis e interpretación de cada uno de los ejes problemáticos. La exposición final de resultados se guía por los hallazgos y conclusiones de la interpretación.

En todos los casos, los resultados de una sistematización o investigación deben ser socializados a través de diferentes medios, según los colectivos sociales a los que se les quiere hacer llegar. En primer lugar, al conjunto de integrantes de la organización, que si bien es cierto han participado en los diferentes momentos del proceso y ha venido enterándose de los avances, son los primeros interesados en conocer los resultados globales; en segundo lugar, a otros colectivos que trabajan en el mismo territorio o en los mismos campos temáticos; en algunos casos, se busca poner en discusión el conocimiento generado en espacios académicos institucionales.

En nuestra experiencia investigativa hemos acudido a diferentes estrategias y formatos comunicativos. En todos los casos se produce un informe escrito que da cuenta del proceso metodológico vivido, de la reconstrucción descriptiva del proceso y de una interpretación en torno a los ejes problemáticos. Salvo algunas excepciones, dicho 
documento se editó como libro, procurando la mayor claridad y sencillez en el lenguaje. En algunos casos se produjeron materiales de amplia difusión (videos, cartillas, programas radiales). Estos materiales se ponen en discusión a través de la realización de talleres, foros y socializaciones públicas.

En la mayoría de los casos, nuestras investigaciones no terminan con la elaboración y socialización de los resultados. Dado el sentido crítico y el interés práctico de las mismas, se realiza una reunión entre el grupo investigador de la universidad y el colectivo de la organización para hacer un balance evaluativo, en términos políticos, de los conocimientos producidos y de los procesos formativos y de transformación generados por la investigación. En algunas ocasiones, surge la necesidad de realizar nuevas investigaciones, de incorporar cambios en las dinámicas y prácticas de las organizaciones y movimientos o de promover acciones de formación.

\section{In-conclusiones}

Las reflexiones iniciales sobre el pensar crítico desde América Latina, así como los criterios y procesos metodológicos descritos, permiten confirmar que al igual que los demás enfoques investigativos y metodológicos existentes en el campo de los estudios sociales, el nuestro emerge y se configura en unos contextos históricos concretos, que condicionan (no determinan) sus sentidos, estrategias y modos de operar. Así mismo, que los sujetos que agencian estas propuestas, también aportan desde sus particulares intereses y propósitos investigativos. En nuestro caso, la investigación crítica participativa ha procurado devenir articulada a procesos organizativos comunitarios y a movimientos sociales y ha estado marcada por nuestra identificación con la educación popular.

Los criterios investigativos expuestos son una elaboración parcial y aún abierta de una construcción colectiva y a escala latinoamericana en torno a la producción de conocimiento desde y sobre prácticas de transformación social. Por tanto, no tiene una pretensión universalista ni busca deslegitimar otras prácticas investigativas actualmente presentes dentro y en las márgenes de las ciencias sociales. Expresan un devenir y un 
caminar singular, que ha buscado responder a desafíos de conocimiento gestados desde prácticas organizativas y de acción colectiva, en diálogo con otras propuestas y reflexiones que se asumen como críticas y alternativas.

Por ello, el itinerario de decisiones y acciones metodológicas presentado no es un diseño rígido para ser "aplicado" a cualquier realidad. Es una invitación a que otros colegas con los que compartimos trayectos, opciones y proyectos similares, también se animen a sistematizar y a comunicar sus propias experiencias, elaboraciones y reflexiones.

\section{Referencias Bibliográficas}

AGUILERA, A.; GONZÁLEZ M. I. y TORRES A. (2012). “Investigar subjetividades y formación de sujetos con organizaciones y movimientos sociales", en: Claudia Piedrahita y otros, Acercamientos metodológicos a la subjetivación política: debates latinoamericanos, Buenos Aires, CLACSO

AGUILERA, A.; GONZÁLEZ M. I. y TORRES A. (2015). Reinventando la comunidad y la política: formación de subjetividades, sentidos de comunidad y alternativas políticas en procesos alternativos locales. Bogotá, Universidad Pedagógica Nacional

CASTORIADIS, C. (1997). Ontología de la creación. Ensayo y error, Bogotá

BARRAGÁN, D.; MENDOZA, C.; TORRES, A., (2006). "Aquí todo es educativo. Saberes pedagógicos y prácticas formativas en organizaciones populares”, en: Revista Folios \# 23, Bogotá, Facultad de Humanidades, Universidad Pedagógica Nacional

BARRAGÁN, D. Y TORRES, A. (2017). Sistematización como investigación interpretativa crítica. Bogotá, SINTESIS - El Búho (en prensa)

CARR, W. y KEMMIS, S. (1988). Teoría crítica de la enseñanza. Barcelona, Martínez Roca

CASTRO S. y MENDIETA E. (coordinadores) (1998). Teorías sin disciplina. Latinoamericanismo, poscolonialidad y globalización en debate. USE -Miguel Ángel Porrúa, México D.F.

CENDALES, L., PERESSON, M. y TORRES, A. (1990). Los otros también cuentan. Elementos para una recuperación colectiva de la historia, Bogotá, Dimensión Educativa

CENDALES, L. y TORRES, A., (1991). En los tiempos de adelante. Recuperación histórica y cultural de los resguardos de los Pastos, Bogotá, Diócesis de Ipiales - DIMED 
CENDALES, L. y TORRES, A., (1995). El esfuerzo no fue en vano. Memoria colectiva de COAGROSARARE. Bogotá, Dimensión Educativa

CENDALES, L. y TORRES, A.,, (2001). "Recordar es vivir. Algunas técnicas para reactivar la memoria colectiva", en: Aportes \# 56, Bogotá, Dimensión educativa

CENDALES, L. y TORRES, A., (2001A). Reconstruyendo nuestra historia. Memoria colectiva de los barrios de Armenia. Bogotá, FOREC - Fundación Restrepo Barco

CENDALES, L. y TORRES, A., (2006). "La sistematización como experiencia investigativa y formativa”, en: La Piragua \# 23. Panamá, CEAAL

CUEVAS, P.; NARANJO, J. y TORRES, A. (1996). Discursos, prácticas y actores de la Educación Popular en Colombia, Bogotá, Universidad Pedagógica Nacional

FALS BORDA, O. (1970). Ciencia propia y colonialismo intelectual, Bogotá, Punta de Lanza FALS BORDA, O. (1994). El problema de como investigar la realidad para transformarla por la praxis. Bogotá, Tercer Mundo

FOUCAULT, M. (2006). Sobre la ilustración. Madrid, Tecnos

GROSFOGUEL, R. y ROMERO, J. (2009). Pensar decolonial. Caracas, Editorial La Urbana

HARAWAY, D. (1995). Ciencia, cyborgs y mujeres. La reinvención de la naturaleza, Madrid, Cátedra

IBÁÑEZ, J. (1994). El regreso del sujeto, Madrid, Siglo XXI

IBÁÑEZ, J. (1998). Nuevos avances en investigación social. Proyecto ediciones, Barcelona

JIMÉNEZ A. y TORRES, A. (2004). La práctica investigativa en ciencias sociales, Bogotá, Universidad Pedagógica Nacional

MATO, D. (2005). "Estudios y otras prácticas intelectuales en cultura y poder". En: Mato Daniel (compilador). Cultura, política y sociedad. FLACSO, Buenos Aires

MENDOZA, C. y TORRES, A. (2011). "La sistematización de experiencias. Presupuestos epistemológicos y procesos metodológicos”. En: Páramo Pablo (coord.) La investigación en ciencias sociales: estrategias de investigación. Universidad. Piloto de Colombia, Bogotá

PORTELI, H. (1985). Gramsci y el bloque histórico. México, Siglo XXI editores

PUERTA, J. (2015). Interpretar el horizonte. Caracas, CELARG

RIVERA, S., compiladora (1997). Debates post-coloniales. Una introducción a los estudios de la subalternidad. La Paz, Sephis - Aruwiyiri

ROIG, A. (2004). Teoría y práctica del pensamiento latinoamericano. México, Fondo de Cultura Latinoamericano 
SANCHEZ, I. y SOSA, R. (coordinadoras) (2004). América Latina: los desafíos del pensamiento crítico. UNAM - Siglo XXI, México D.F.

SANTOS, B. de S. (2006). Renovar la teoría crítica y reinventar la emancipación social. CLACSO, Buenos Aires

SANTOS, B de S. (2013). Descolonizar el saber, reinventar el poder. Santiago de Chile, LOM SCOTT, J. (2000). Los dominados y el arte de la resistencia. Editorial Era, México D.F.

TORRES, A. (1992). Organizaciones barriales y educación popular. Neiva, Escuela Popular Claretiana

TORRES, A. (1993). La ciudad en la sombra. Barrios y luchas Populares en Bogotá. Bogotá, CINEP

TORRES, A. (1994). Iniciación a la investigación histórica. Bogotá. Universidad Santo Tomás

TORRES, A. (1994a). "Recuperando la historia desde abajo. Enfoque y cuestiones metodológicas”, en: Cuadernos de filosofía latinoamericana, Bogotá, USTA

TORRES, A. (1995), Aprender a investigar en comunidad. UNISUR. Bogotá, 1995

TORRES, A. (1996), Enfoques cualitativos y participativos de investigación social, Bogotá, UNAD

TORRES, A. (1996무), “La sistematización desde la perspectiva interpretativa”, en: Aportes \# 44. Bogotá, Dimensión Educativa

TORRES, A. (1998), Estrategias y técnicas de investigación cualitativa. Bogotá, UNAD

TORRES, A. (1999), "La sistematización de experiencias educativas. Reflexiones sobre una práctica reciente”, en Pedagogía y Saberes. № 13, Universidad Pedagógica Nacional (2000), "Las complejidades de lo social y los desafíos a la investigación crítica", en: Cuadernos de sociología \# 35, Bogotá, Revista de Sociología de la Universidad Santo Tomás

TORRES, A. (2003). "Pasados hegemónicos, historias populares e historias subalternas", en: Catherine Walsh (editora), Estudios culturales latinoamericanos en y desde el Área Andina, Quito, UASB - Abya yala

TORRES, A. (2004). “Por una investigación desde el margen”, en: Jiménez y Torres, La práctica investigativa en Ciencias Sociales, UPN, Bogotá

TORRES, A. (2004a). “Sistematización de experiencias de organización popular”. En: Aportes \# 57. Bogotá, Dimensión Educativa 
TORRES, A.(2006). Paz joven. La participación en los procesos de construcción de paz, Manizales, ACDI/Plan Internacional/CINDE

TORRES, A. (2007). Identidad y política de la acción colectiva. Organizaciones populares y luchas urbanas en Bogotá 1980 -2000, Bogotá, Universidad Pedagógica Nacional

TORRES, A. (2008). “Investigar en los márgenes de las Ciencias Sociales”, en: Folios \# 27, Bogotá, Revisa de la Facultad de Humanidades. Universidad Pedagógica Nacional

TORRES, A. (2008a $)$. "Rehaciendo la memoria de las organizaciones populares", en: Adrián Serna (compilador), Memoria y conflicto, Bogotá, Universidad Distrital Francisco José de Caldas. Bogotá

TORRES, A. (2010). "From participatory research to the systematization of experiences", en: International Journal of Action Research \# 6. Mering, Germany

TORRES, A. (2011). “Límites y márgenes de las ciencias sociales”, en: Esfera. Vol 1, Bogotá

TORRES, A. (2014). "Producción de conocimiento desde la investigación crítica”, en: Nómadas \# 40, Bogotá, IESCO - Universidad Central

TORRES, A. y NARANJO, J. (1996). Marcando huellas, forjando futuro. Historia del Jardín Infantil Comunitario de Ciudad Hunza. Bogotá, Dimensión Educativa.

TORRES, A. y TORRES, A., coordinadores. (2011), Sujetos, prácticas y sentidos de la participación local en Bogotá. Bogotá, MGU - UPC.

TORRES, A. y otros (2003). Organizaciones populares, identidades locales y ciudadanía en Bogotá. Bogotá, Universidad Pedagógica Nacional - COLCIENCIAS

USECHE, O. (2016). “Pensamiento crítico y subjetividades de resistencia”, en: Piedrahita Claudia, Díaz, Álvaro y Vommaro, Pablo, Pensamientos críticos contemporáneos: análisis desde Latinoamérica.

VALIENTE, S., GODFRID, J. y BERTEA J. (2017). “Transitando los márgenes. Hacia una investigación de borde”. En: Huellas \# 102, Barranquilla, Universidad del Norte.

WALZER, M. (1993). Interpretación y crítica social. Ediciones Nueva Visión, Buenos Aires ZEMELMAN, H. (1987). Uso crítico de la teoría. El Colegio de México, México D.F.

ZEMELMAN, H. (1992). Los horizontes de la razón. Dialéctica y apropiación del presente (2 vols.). Anthropos y El Colegio de México, Barcelona

ZEMELMAN, H. (1994). "Racionalidad y ciencias sociales”. En: Suplementos \# 45, Anthropos, Barcelona 
ZEMELMAN, H. (2011), “Pensar teórico y pensar epistémico”, en: Irene Sánchez y Raquel Sosa (coordinadoras), América Latina: los desafíos del pensamiento crítico, México DF, UNAM - Siglo XXI. 